\title{
Explotación de mantos de poco espesor, aplicando el método Cámaras y Pilares con Galería Central y Pilares escalonados - Mina Aztlán 2 - Jalisco - México
}

\author{
Exploitation of thin mantles, applying the method Chambers and Pillars with \\ Central Gallery and Stepped Pillars - Mina Aztlan 2 - Jalisco - Mexico
}

\author{
Luís Alberto Arce Gonzáles ${ }^{1}$, Enrique Guadalupe Gómez ${ }^{2}$ \\ Recibido: 01/07/2020 - Aprobado: 30/10/2020 - Publicado: 30/11/2020
}

\begin{abstract}
RESUMEN
En minería subterránea la explotación de mantos de espesores menores a $1.20 \mathrm{~m}$, se torna difícil y muy a menudo su costo de minado es elevado, esto debido al método de explotación tradicional que se suele emplear. Este trabajo tiene como objetivo plantear un nuevo diseño de explotación y dar solución al problema operativo en la unidad minera de Aztlán 2 en Autlán de Navarro, Jalisco, México, donde el manto tiene un espesor menor de $1.20 \mathrm{~m}$. Para alcanzar el objetivo planteado se diseñó una variante del método cámara y pilares, que se le ha denominado: cámaras y pilares con galería central y pilares escalonados o sencillamente T Room, permitiendo reducir el costo de minado de USS $\$ 59.00$ dólares la tonelada extraída a USS $\$ 38.00$ dólares, además le da mayor velocidad a la explotación y pasar la producción de 200 toneladas /mes en forma artesanal a 3400 $\mathrm{Tn} / \mathrm{mes}$, usando esta nueva variante. La aplicación de esta variante muestra que es económicamente viable para estructuras que tengan similares características, es decir tengan cajas competentes y cuyos espesores sean menores a $1.2 \mathrm{~m}$.
\end{abstract}

Palabras clave: Cámaras y pilares; pilares escalonados; galería central de explotación.

\begin{abstract}
In underground mining, the exploitation of mantles of thicknesses less than $1.20 \mathrm{~m}$, becomes difficult and very often its cost of mining is high, this due to the traditional exploitation method that is usually used. This work aims to propose a new exploitation design and provide a solution to the operational problem in the Aztlan 2 Mining Unit in Autlán de Navarro - Jalisco Mexico, where the mantle with manganese mineral content is less than $1.20 \mathrm{~m}$ thick. For which a variant of the camera and pillars method was designed, which has been called: cameras and pillars with central gallery and stepped pillars or simply T Room, allowing to reduce the cost of mining of US $\$ 59.00$ dollars the ton extracted at US $\$ 38.00$ dollars, also gives greater speed to the exploitation and pass production of 200 Tons / month in a traditional way to 3400 tons / month, using this new variant. The application of this variant shows that it is economically viable for structures that have similar characteristics, that is to say they have competent boxes and whose thicknesses are less than $1.2 \mathrm{~m}$.
\end{abstract}

Keywords: Chambers and pillars; staggered pillars; central exploitation gallery.

1 Universidad Nacional Mayor de San Marcos, Facultad de Ingeniería Geológica, Minera, Metalúrgica y Geográfica. Lima, Perú. Autor para correspondencia: rockblue27@yahoo.com ORCID: https://orcid.org/0000-0002-4421-2000

2 Universidad Nacional Mayor de San Marcos, Facultad de Ingeniería Geológica, Minera, Metalúrgica y Geográfica. Lima, Perú. E-mail: eguadalupeg@unmsm.edu.pe ORCID: https://orcid.org/0000-0001-9583-8807 


\section{INTRODUCCIÓN}

En minería subterránea convencional cuando se tiene mantos de potencias menores a $2.00 \mathrm{~m}$, con rocas encajonantes y mineral de tipo III A, el método elegido es cámaras y pilares; sin embargo, cuando la estructura se reduce a menos de $1.20 \mathrm{~m}$, seguir utilizando la misma metodología, significa un gasto innecesario o excesivo, esto debido que de todas maneras debe de abrirse una sección no menor de 1.80 a $2.00 \mathrm{~m}$ de altura, este caso se presenta en la unidad minera Aztlán 2.

\subsection{Antecedentes}

El lote minero Aztlán 2 fue estudiado en el año 1998 por el ingeniero geólogo Virginio Salgado Terán, determinando recursos geológicos por 684,334 toneladas con una ley media de manganeso de $41 \%$, valorizándolo en US \$ 70'142,500.00 dólares. En el año 2008 realiza un estudio preliminar, para la puesta en operación del lote minero, estimando una inversión de 13 millones de pesos para la puesta en operación del lote minero Aztlán 2.

El Servicio Geológico Mexicano (SGM) (Sociedad Geológica Mexicana, 2007), realiza un estudio de asesoría geológica del lote minero Aztlán 2, concluyen que se tienen 19,840 toneladas de mineral como recursos inferidos, con una ley media de $42.66 \%$, recomendando que realicen primeramente trabajos de taladros diamantinos en superficie, para asegurar la continuidad del manto y proceder al inicio del reacondicionamiento dicha unidad.

\subsection{Ubicación}

La mina está Ubicada en el municipio de Autlán de Navarro (Instituto de Monterrey, 2013), Jalisco, en la carretera libre de Guadalajara a Manzanillo en la ranchería San Francisco (Figura 1).

\subsection{Geología local}

Localmente afloran rocas de una secuencia volcano sedimentaria constituidas por tobas riolíticas y andesíticas de tonalidades rosáceas y zonificaciones de silicificación, se observan intercalaciones muy locales de arenisca y limolita, estas rocas se encuentran afectadas por un intrusivo de composición granodiorítica, el cual aflora en la porción Oeste del área de estudio.

\subsection{Controles de mineralización}

El yacimiento de manganeso en la zona de Autlán está formado por óxidos y silicatos que se presentan en forma de manto, encajonado en un cuerpo de tobas riolíticas endurecidas, las principales especies mineralógicas en las que se presenta son en orden de importancia: braunita, minerales del grupo del psilomelano y pirolusita, además se presentan como minerales accesorios, barita, calcita, óxidos de hierro, cuarzo y minerales calcosilicatos de magnesio.

En general, el manto tiene un comportamiento estructural homogéneo, presenta una inclinación de tan solo $5^{\circ}$ y su rumbo del echado es de $45^{\circ}$ al SE, el espesor observado fue en cierta medida también uniforme, fluctuando entre 1.00 y 1.20 metros.

\subsection{Características del mineral}

Como se menciona en los párrafos anteriores el mineral económico lo componen óxidos de manganeso, especialmente braunita el cual macroscópicamente presenta una forma bandeada, compacta, de alto peso específico (4.8) con una dureza de 6.5 según la escala de Mohs, además de gran resistencia a la compresión uniaxial. (110 Mpa), su color es pardo oscuro y brillo semi metálico (Figura 2).

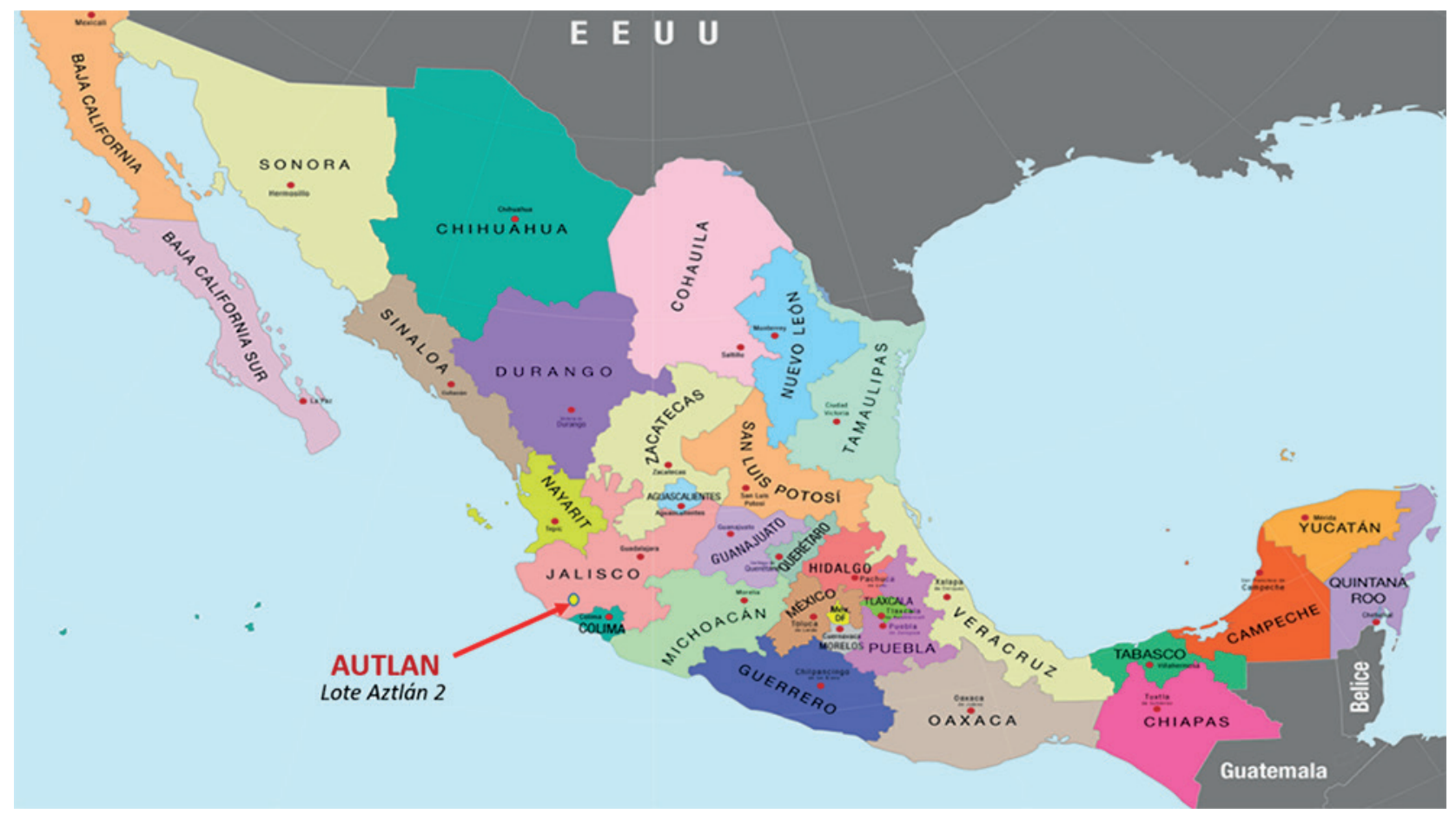

Figura 1. Plano de Ubicación del Lote Aztlán 2 en Autlán, Estado de Jalisco, País México. 


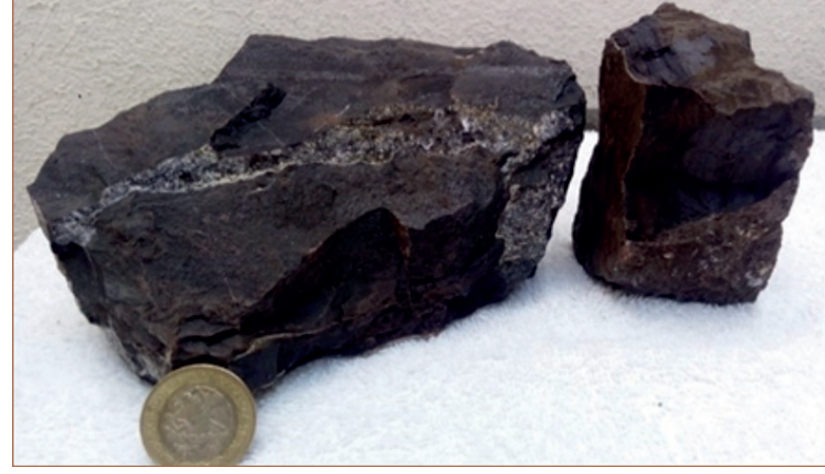

Figura 2. Muestras de mineral de manganeso que corresponden mayoritariamente a braunita el cual es un nesosilicato de manganeso. $\mathrm{Mn}^{2+} \mathrm{Mn}_{6}^{3+} \mathrm{SiO}_{12}$

\subsection{Métodos convencionales}

\subsubsection{Cámaras y pilares convencional}

Este método es utilizado en mantos horizontales a subhorizontales no mayor a 30 grados de buzamiento, con potencias menores a los 2 metros, en cajas competentes. La perforación es neumática y la limpieza se realiza utilizando winche de arrastres, las dimensiones de la cámara y pilares lo determina el cálculo geomecánico basados en los parámetros de la calidad de la roca (Figura 3). Mientras, más grandes sean los esfuerzos al cual es sometida la cámara de explotación, los pilares de soporte son de mayor sección o la cámara de explotación se reduce; pero con ello se reduce también la recuperación del mineral.

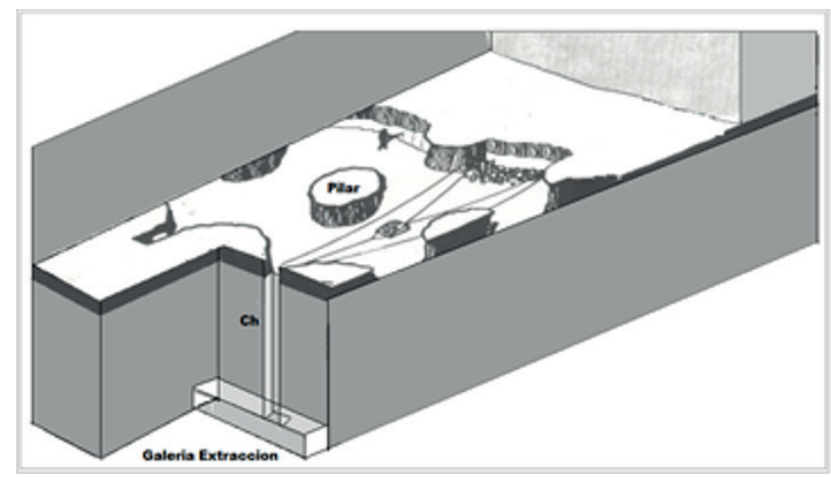

Figura 3. Se grafica la forma como se explota el mineral en mantos de potencia reducida y la disposición de los pilares. Fuente: Herrera \& Gómez (2007)

1.6.2 Cámaras vacías y uso de gatas hidráulicas en retirada

Esta variación del método se aplica en mantos de minerales blandos, especialmente carbón. Este método consiste en sostener la abertura de la cámara de explotación con gatas hidráulicas, en línea y se avanza con ellas a medida que se corta el mineral, la roca caja que va quedando detrás, se hunde y ocupa el espacio abierto, esto modifica la superficie por tanto su aplicación se reduce a yacimientos en zonas donde no se tenga inconveniente con la modificación de la superficie (Figura 4).

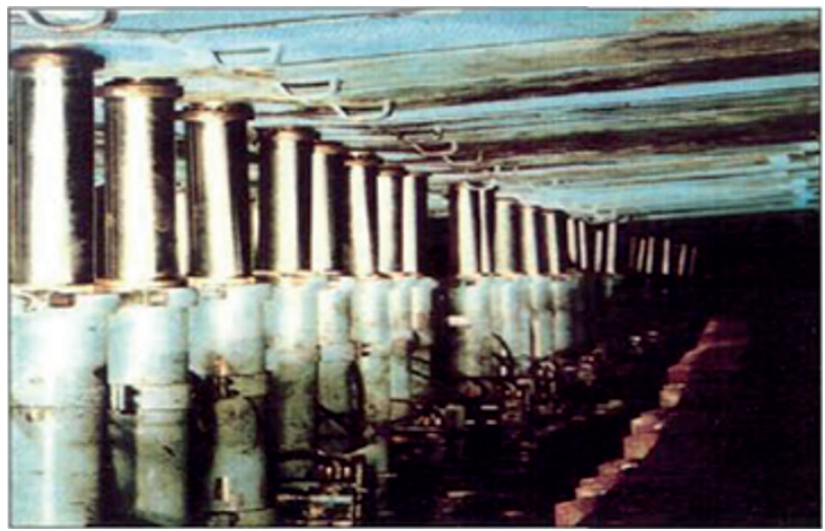

Figura 4. Método cámaras vacías y uso de gatas hidráulicas en retirada, método empleado en mantos de carbón. Fuente: Herrera \& Gómez (2007)

\section{MÉTODOS Y MATERIALES}

El diseño de la investigación desarrollada es de tipo aplicativo porque se aplica una nueva variante de explotación de cámaras y pilares, también es exploratorio, porque es un tema nuevo, descriptivo y para complementar se compara el método convencional con el método propuesto de cámaras y pilares con galería central de explotación y pilares escalonados.

La primera fase de campo se inició con la visita a la unidad minera, haciendo un reconocimiento geológico y minero del yacimiento en estudio, recabando datos de las unidades periféricas que estaban en operación en forma artesanal, se recolectaron muestras dentro del yacimiento, lo cual fue importante para la determinación mineralógica, ya que existen muchos minerales de manganeso.

Como instrumento para la caracterización mineralógica y petrológica, se usó un microscopio de polarización de luz reflejada marca Leitz Ortholux II del laboratorio de Ingeniería Geológica de la Universidad Nacional Mayor de San Marcos-UNMSM, también se midió la resistencia a la compresión uniaxial de muestras de mineral y roca.

Para los distintos puntos de observación se utilizó un GPS portátil Garmin, además se hizo diversas mediciones de las secciones de las labores artesanales de cámaras y pilares utilizando flexómetro, midiéndose en promedio pilares redondos de 2.5 metro de diámetro y cámaras de $5.5 \mathrm{~m}$ de ancho y $2.0 \mathrm{~m}$ de alto. La estructura presenta una potencia media de $0.80 \mathrm{~m}$.

\section{RESULTADOS}

\subsection{Explotación convencional del manto en lotes periféricos}

Desde los inicios de la explotación del manto mineralizado, se usa el método de cámaras y pilares convencional, fueron los primeros dueños que iniciaron la explotación, desde los años 50. Dicha explotación se realizó en sectores donde el manto presentaba una potencia media de 1.20 a $1,80 \mathrm{~m}$ con cámaras de $5.00 \mathrm{~m}$ y pilares de $3,00 \mathrm{~m}$ de forma cuadrada 
o circular, toda la actividad era convencional y el mineral se acarreaba con una locomotora en los niveles inferiores horizontales, luego en un punto el convoy ascendía a superficie por un inclinado.

A medida que la potencia del manto se fue reduciendo a una media de 0.80 a $1,00 \mathrm{~m}$, el método de cámaras y pilares se tornó más costoso, debido que se continuaba abriendo toda la cámara para extraer el mineral con poco espesor, lo cual generaba una alta dilución por la mayor cantidad de estéril que se rompía al generar la cámara, o por el doble ciclo de perforación y voladura primero se extrae el estéril y luego el mineral.

\subsubsection{Costos de operación}

La pequeña unidad minera Aztlan 2, ha estado realizando explotación artesanal, en la periferia de sus linderos, en los lotes pertenecientes a la empresa minera Autlán, bajo un convenio, en el cual la producción se lo vendía a dicha empresa a un valor de US \$ $\mathbf{6 5 . 1 0}$ dólares/tonelada, para una ley mínima de $30 \%$ de óxidos de manganeso, la producción que se obtenía era de 200 toneladas de mineral por mes.

El costo de operación en la explotación artesanal que se realiza es de aproximadamente US \$ 59.16 dólares / tonelada, esto es mientras se trabaje sin contratiempos; sin embargo, se tienen paradas muy frecuentes por fallas de los equipos y otros.

\begin{tabular}{lr} 
Distribución de los costos: & US \$ /TM \\
- Rotura mineral & 21.34 \\
- Preparaciones & 9.03 \\
- Desmonte & 21.23 \\
- Acarreo & 3.44 \\
- Pallaqueo & 2.62 \\
- Permisos & 1.50 \\
\cline { 2 - 2 } Total: & US \$ $\mathbf{5 9 . 1 6}$
\end{tabular}

Total:

US \$ 59.16

El precio de compra que la empresa minera Autlán S.A. de C.V, brinda a los dueños de la concesión Aztlan 2, es de US \$ $\mathbf{6 5 . 1 0}$ dólares la tonelada de mineral extraído, puesto en cancha.

Una buena posibilidad de acceder a la concesión Aztlan 2 para explotar su mineral es gracias a los convenios de servidumbre que se tiene con los propietarios de las concesiones periféricas, utilizando labores antiguas (un túnel de $700 \mathrm{~m}$, galerías y chimeneas)

\subsection{Nuevo método de explotación de cámaras y pilares con galería central de explotación y pilares escalonados para mantos de potencias reducidas}

El método cámaras y pilares con galería central de explotación y pilares escalonados o $T$ Room, es una variación del método cámaras y pilares, el cual se diseñó para ser utilizado especialmente en mantos de poco espesor en una secuencia de explotación.

\subsubsection{Galería central}

Consiste en realizar una galería al centro, donde será la cámara de explotación con una sección que permita la circulación de equipos de limpieza (scooptram de 1.5 a 3,2 $\mathrm{Yd}^{3}$ ) con una altura de trabajo para la perforadora jackleg para barra de 8 pies (Sección 2.5 x 2.2 m). En la Figura 5 , se muestra una malla sugerida de perforación para la construcción de la galería central.

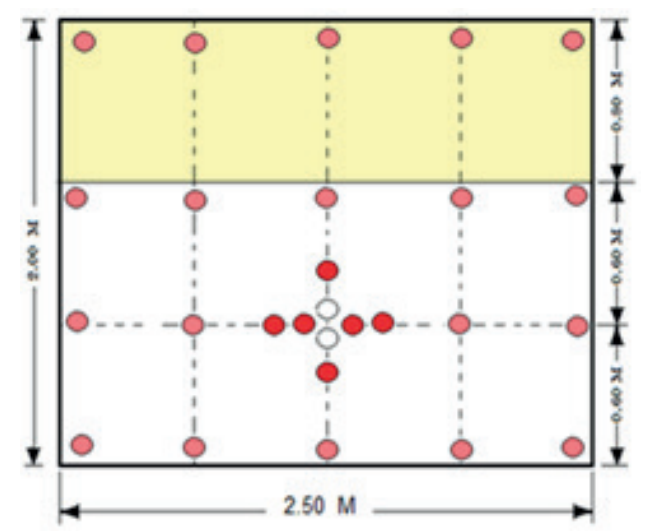

Figura 5. Malla sugerida, obsérvese que la voladura del estéril sale como un frente y la voladura del mineral sale en breasting

La galería excavada será utilizada para realizar la perforación, voladura y limpieza del mineral roto de la cámara y corresponde al laboreo de preparación del método. Esta preparación se realiza ubicando el mineral en la caja techo y el resto de la sección será en estéril. La Figura 6 muestra la posición de esta labor central. Para evitar diluir el mineral se recomienda disparar el estéril y posteriormente el mineral.

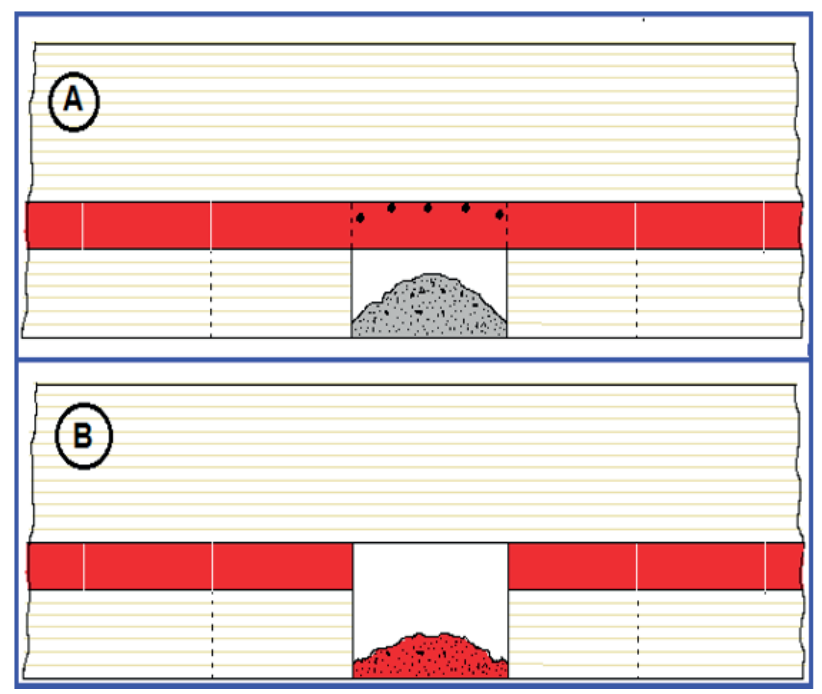

Figura 6. Construcción de la galería central de explotación, en A se muestra la rotura del estéril y en $B$ la rotura del mineral.

\subsubsection{Explotación del tajeo.}

Una vez realizada la preparación de la galería central que atraviesa las cámaras se procede a la explotación del manto. 


\section{Perforación}

Desde la galería central se posesiona, la perforadora haciendo un ángulo con la horizontal y la barra, de aproximadamente 15 a 20 grados, esto para darle una fuerza de salida al material cuando se dispare (patada), la figura 7 muestra este procedimiento.

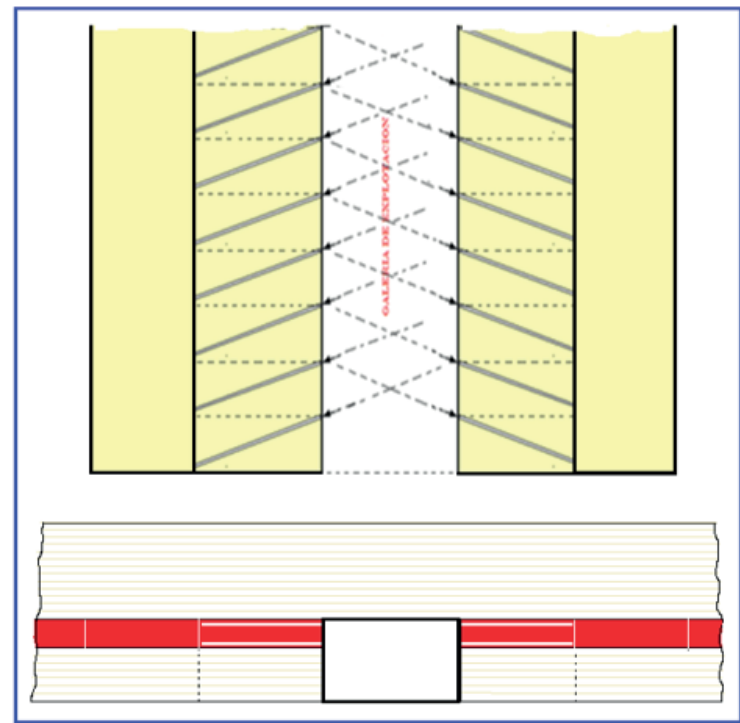

Figura 7. Muestra la manera como se disponen los taladros al realizar la perforación, el ángulo de los taladros permitirá que el mineral roto se deposite en la galería central.

Luego de la perforación y voladura en el tajeo, el mineral roto caerá dentro de la galería de explotación central y lo que quede entre la banca de estéril se jala con la ayuda de una lampa tipo rastrillo de mango largo, manualmente hacia esta labor, donde el equipo de limpieza lo llevará al pozo (Figura 7).

\section{Limpieza y acarreo del mineral}

El mineral roto caerá dentro de la galería de explotación central y lo que quede entre la banca de estéril, se jala con la ayuda de una lampa tipo rastrillo de mango largo, manualmente hacia esta labor, un equipo de limpieza (scooptram $1.5 \mathrm{Yd}^{3}$ ) lo llevará al pozo, desde donde un vehículo tipo mini dumper recogerá el mineral de la tolva y lo llevara a superficie. En la Figura 8 se observa cómo queda la labor luego de la voladura.

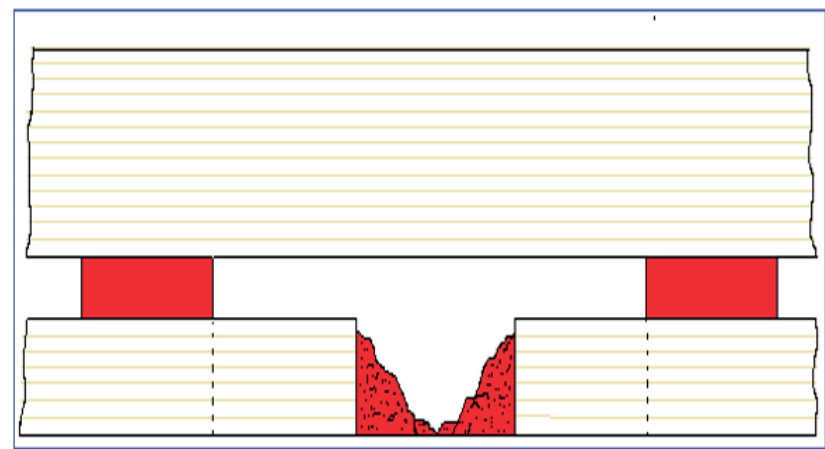

Figura 8. Obsérvese que el mineral queda en la galería central de explotación donde está listo para ser extraído por un scoop y conducido a la chimenea y de esta se acarrea a superficie por un mini dumper
3.2.3 Diseño y cálculo de los pilares, usando método del área de tributaria

Determinación de la carga que soporta el pilar

Resistencia a la compresión uniaxial

$$
\mathrm{Sc}=120,000 \mathrm{Kpa}
$$

Diámetro de probeta $\mathrm{D}=54 \mathrm{~mm}$

Determinación de la constante K

$$
\begin{aligned}
& \mathrm{K}=\mathrm{Sc} \sqrt{D} \\
& \mathrm{~K}=120000 \sqrt{0,054} \\
& \mathbf{K}=\mathbf{2 7 8 8 5 . 4 8}
\end{aligned}
$$

Calculamos la tensión del pilar (Sp) usando la fórmula de Bieniaswki

$$
\begin{aligned}
& \mathrm{Sp}=\mathrm{S} 1(0.64+0.36 \mathrm{~W} / \mathrm{h}) \\
& \mathrm{S}_{1}=\mathrm{K} / \sqrt{0}, 9144 \\
& \mathbf{S}_{1}=\mathbf{2 9 1 6 1 . 5 1}
\end{aligned}
$$

\section{Datos:}

Ancho del pilar $(\mathrm{w})=2.00 \mathrm{~m}$

Potencia mineral $(\mathrm{h})=0.8 \mathrm{~m}$

$$
\begin{aligned}
& \mathrm{Sp}=\mathbf{S}_{\mathbf{1}}(0.64+0.36(2.0 / 0.8)) \\
& \mathbf{S p}=\mathbf{4 4 9 0 8 . 7 2 5 4} \mathbf{K p a}
\end{aligned}
$$

Cálculo de la carga que soporta el pilar (Sp)

Sp: La tensión del pilar (Kpa)

$\mathrm{H}$ : Profundidad de la capa del mineral (m) 65

W: Ancho del pilar (m) 2.0

L: $\quad$ Longitud del pilar (m) 2.0

B: Ancho de la cámara $(\mathrm{m}) \mathbf{6 . 5 0}$

g: Densidad de la roca 2,5 $\mathbf{T n} / \mathbf{M}^{3}$

g: Aceleración de la gravedad $9.81 / \mathrm{S}^{2}$ (Figura 9)

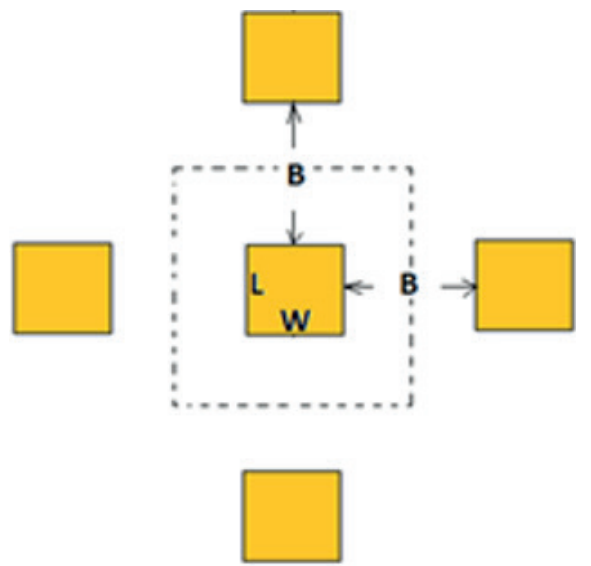

Figura 9. El grafico muestra la longitud del pilar L, el ancho del pilar W y el ancho de la cámara $B$ 
Fórmula para secciones cuadradas

$$
\begin{aligned}
& \mathrm{Sp}=\mathrm{g} \mathbf{g} \mathrm{H}\left[(\mathrm{W}+\mathrm{B})^{2} / \mathrm{W}^{2}\right] \\
& \mathrm{Sp}=24.525(65)\left[\left(2.0+6.50^{2} / 2.0^{2}\right]\right. \\
& \mathrm{Sp}=\mathbf{2 8 , 7 9 3 . 8 8 2 8} \mathbf{K p a} .
\end{aligned}
$$

Factor de seguridad

$$
\begin{aligned}
& \mathrm{F}=\mathrm{Sp} / \mathrm{Sp} \\
& \mathrm{F}=44,908.7254 / 28,793.8828 \\
& \mathbf{F}=\mathbf{1 , 5 6}
\end{aligned}
$$

\section{Recuperación (e)}

$$
\begin{aligned}
& \mathrm{e}=1-[\mathrm{W} /(\mathrm{W}+\mathrm{B}) \times \mathrm{L} /(\mathrm{L}+\mathrm{B})] \\
& \mathrm{e}=1-[2.0 /(2.0+6.5) \times 2.0 /(2.0+6.5)] \\
& \mathbf{e}=\mathbf{9 4} \%
\end{aligned}
$$

3.2.4 Corrección de pilares usando formula de María José Yrarrazaval Herrera

Ajuste de los parámetros de pilares utilizando la fórmula de Yrarrazaval Herrera (2013)

$$
\left.S p=\operatorname{UCS}_{50}\left(\frac{(\operatorname{RMR}-100}{\mathrm{exp}}\right)\right)^{D}\left(A+B \frac{W}{H}\right)^{E}\left(\frac{0.05}{W}\right)^{F}
$$

$\mathrm{Sp}$ : Resistencia del pilar o testigos en Mpa

UCS: Resistencia a la compresión uniaxial de

\begin{tabular}{|c|c|}
\hline \multicolumn{2}{|c|}{$\begin{array}{c}\text { Valores de los parametros } \\
\text { de Ajuste MI }\end{array}$} \\
\hline PARAMETRO & VALOR \\
\hline A & 0.75 \\
\hline B & 0.5 \\
\hline C & 11.6 \\
\hline D & 0.62 \\
\hline$E$ & 1.04 \\
\hline $\mathrm{F}$ & 0.12 \\
\hline
\end{tabular}
la roca intacta $120 \mathrm{Mpa}$

We: $\quad$ Ancho efectivo del pilar o testigo según Esterhuisen (m)

$\mathrm{H}$ :

$$
\text { Altura del pilar o testigo (m) } 0.80
$$

RMR: rocoso 73

Calificación de la calidad del macizo

A, B, C, D, E, F: Seis constantes de ajuste (Figura 10)

Figura 10. Valores de ajuste de la fórmula de Yrarrazaval Herrera (2013)

El ancho efectivo (We) está relacionado al efecto del largo del pilar, por lo que según la fórmula de Esterhuisen, se aprecia que la relación $\mathrm{W} / \mathrm{H}$ es mayor a 1.4 entonces no se usa, pasando a usarse la fórmula de Wagner, siendo la siguiente:
$\mathrm{W} \mathrm{e}=4$ (área del pilar / perímetro del pilar)

$$
\mathrm{We}=4(2.0 \times 2.0 / 8)
$$$$
\text { We }=2.0 \mathrm{~m} *
$$

* Se aprecia que la relación del largo del pilar, por ser de sección cuadrada no afecta el valor final, siendo el ancho efectivo el mismo al propuesto inicialmente.

$\mathrm{Sp}=120\left(e^{(73-100) / 11.6) 0.6}\left(0.75+0.50\left(\frac{2.0}{0.8}\right)^{1.04}(0.05 / 2.0)^{0.12}\right.\right.$

$\mathrm{Sp}=62.2388 \mathrm{Mpa}=62,238.8 \mathrm{Kpa}$

Cálculo de la carga del pilar

Utilizamos el ancho efectivo (We) del pilar al realizar los cálculos de la carga sobre el pilar

$$
\mathbf{S p}=\boldsymbol{q} \mathbf{g} H\left[\frac{(\mathrm{W}+\mathrm{B})^{2}}{\mathrm{~W}^{2}}\right]
$$

$\mathrm{Sp}=28,793.8828 \mathrm{Kpa}$

Factor de seguridad

$$
\begin{aligned}
& \mathbf{F}=\mathbf{S p} / \mathrm{Sp} \\
& \mathbf{F}=\mathbf{6 2 , 2 3 8 . 8} / 28,793.8828 \\
& \mathbf{F}=\mathbf{2 . 1 6}
\end{aligned}
$$

$\underline{\text { Recuperación (e) }}$

$$
\begin{aligned}
& \mathbf{e}=1-(\mathrm{W} /(\mathrm{W}+\mathrm{B}))(\mathrm{L} /(\mathrm{L}+\mathrm{B})) \\
& \mathbf{e}=1-(2,0 /(2,0+6,5))(2,0 /(2,0+6,5)) \\
& \mathbf{e}=\mathbf{9 4} \%
\end{aligned}
$$

\subsubsection{Simulación de esfuerzos por elementos finitos}

Tomando en cuenta las observaciones de campo se consideró las propiedades tanto del mineral como las de la roca encajonante, que se acerca a los parámetros mostrados en la simulación en cuanto al factor de seguridad (Figura 11).

\subsubsection{Costos}

Se realizaron los cálculos de los costos que se tendría en una operación de esta unidad para una producción inicial aproximada de 3400 toneladas por mes.

En esta operación se perforaría con jackleg con barra de 8 pies y la limpieza se realizaría con un scooptram de 1.5 Yardas cubicas, el acarreo con mini dumper de $0.75 \mathrm{M} 3$ aproximadamente, de capacidad de carga, para un recorrido de 700 metros.

El método de explotación cámaras y pilares con galería central y pilar escalonado, como se ha explicado, una de las ventajas que se aprecia del método es que no se extrae el estéril de la cámara, cuando se explota mineral, este desmonte equivale a $\mathbf{5 2 , 8} \mathbf{~ m 3 / c a ́ m a r a , ~ l o s ~ c o s t o s ~ u n i t a r i o s ~}$ en los ciclos de este método son los siguientes: 


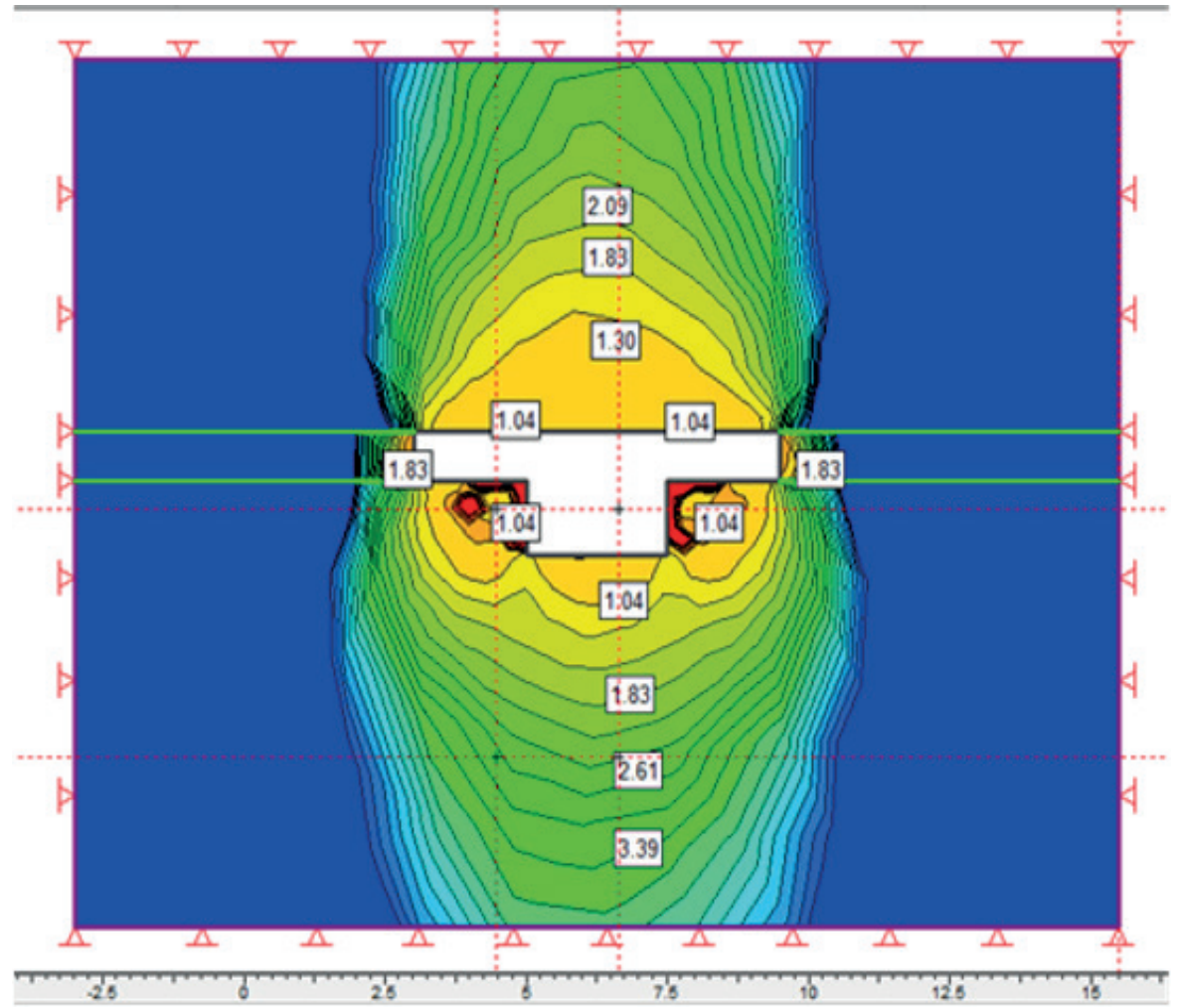

Figura 11. Análisis de factor de seguridad (abierto total luego de explotación)

- $\quad$ Rotura de Mineral

US \$ TM

- Preparaciones

14.10

18.47

2.60

1.35

0.09

0.24

1.50

38.35

\section{COSTO Total}

Estos costos han sido calculados para una producción aproximada de $3400 \mathrm{Tn}$ aproximadamente por mes, las cuales pueden incrementarse a medida que la mina adquiera capital propio y pueda financiar una segunda inversión para duplicar su producción.

\subsubsection{Variaciones del nuevo método}

El método es sencillo para su aplicación en mantos menores a $1.20 \mathrm{~m}$ y con roca encajonante competente, puede variar sus ciclos convencionales, el cálculo que se ha realizado es para una explotación donde la perforación es convencional, y se utiliza perforación neumática, taladros de 8 pies y la limpieza se realiza con un micro scoop de 1.5 Ydas3, pudiendo usarse hasta uno de 3.2 Ydas 3 con la sección de $2.5 \times 2.2 \mathrm{~m}$ de la galería central de explotación.

Una variante más del método puede ser con taladros largos esto debido que actualmente se cuenta en el mercado con equipos de perforación mecanizada de taladros largos para secciones reducidas que pueden ser aplicados a mantos de potencias menores a $1.20 \mathrm{~m}$ lo cual aumentaría la productividad, El aumento de las dimensiones de la cámara puede generar problemas en el auto soporte, por lo que el empernado en la zona central de la cámara (galería de explotación), reduce el riesgo, además la colocación de puntales en la sección media del área de explotación reduciría el esfuerzo generado en el techo a parámetros seguros.

\section{DISCUSIONES}

En cuanto a discusión de resultados, tenemos el depósito Santa Rosa (Mn-Ba) que se encuentra cerca al poblado de San Nicolás, México, consiste en una veta-brecha de óxidos de manganeso de $500 \mathrm{~m}$ de longitud con inclinaciones subverticales, con óxidos de manganeso (romanechita y pirolusita), barita, ópalo, cuarzo, calcita, magnetita maghemita y goethita (Rodríguez-Díaz et al., 2008). En cambio, en el yacimiento de manganeso en la zona de Autlán, está conformado por óxidos y silicatos que se presentan en forma de manto, las principales especies mineralógicas en las que se presenta son en orden de importancia: braunita, minerales del grupo del psilomelano y pirolusita, además se presentan como minerales accesorios, barita, calcita, óxidos de hierro, cuarzo y minerales calcosilicatos de magnesio.

El proyecto minero Mar y Cielo (Vilches Vera, 2015) ubicado en Chile, tiene como objeto la explotación de mineral de manganeso a cielo abierto con una tasa de extracción del orden de 220.000 toneladas de mineral con manganeso por mes en cambio en Autlán la extracción es subterránea con cámaras y pilares convencional y mejorado con cámaras y pilares con galería central y pilares escalonados, proyecta solo una producción de 3400 TM. 


\section{CONCLUSIONES}

- El nuevo método de minado denominado cámaras y pilares con galería central de explotación y pilares escalonados, permite una explotación racional y ordenada, de los recursos en estructuras angostas inclusive menores a un metro, extrayéndose esencialmente la zona del mineral y dejando la estéril in situ, todo ello gracias a la galería central de explotación, desde donde se realiza la perforación y limpieza del mineral roto.

- Se logra perturbar menos el macizo rocoso, esto debido a que las secciones abiertas son reducidas.

- Se reduce el costo de minado, si se compara con el tradicional método de cámaras y pilares, porque no se extrae en cada cámara de explotación $52,8 \mathrm{~m}^{3}$ de estéril.

- Este método puede iniciarse con una producción de 3400 TM. para luego implementar más unidades de perforación y personal e incrementar la producción mensual.

- El costo de minado del método a implementarse en la unidad Aztlan 2 es de US\$ 38.35 dólares por tonelada, siendo mucho más económico que el costo de minado del método convencional artesanal, de US \$59.16 dólares la tonelada, mostrándose una diferencia significativa de US\$ 20.80 dólares / TM. Esto muestra lo económico que puede ser la implementación del método sugerido.

\section{AGRADECIMIENTOS}

Un especial agradecimiento a la Licenciada en Negocios Señorita Martha Villegas, por los contactos realizados para llevar adelante este estudio en Autlán de Navarro, Estado de Jalisco - México, un agradecimiento al Ingeniero Martin Flores Palacios por el apoyo en las simulaciones del método.

\section{REFERENCIAS}

Herrera, H., \& Gómez, J. (2007). Diseño de explotaciones e infraestructuras mineras subterráneas. In Biblioteca de la Universidad Politécnica de Madrid. (Ed.), Universidad Politécnica de Madrid. Departamento de Explotación de Recursos Minerales y Obras Subterráneas. http://oa.upm. es/21841/

Instituto de Monterrey, M. (2013). Compañía Minera Autlán. www.autlan.com.mx

Rodríguez-Díaz, A. A., Blanco-Florido, D., Canet, C., GervillaLinares, F., González-Partida, E., Prol-Ledesma, R. M., Morales-Ruano, S., \& García-Vallès, M. (2008). Metalogenia del depósito de manganeso Santa Rosa, baja California sur, México. Boletin de La Sociedad Geologica Mexicana, 62(1), 141-159. https://doi.org/10.18268/ BSGM2010v62n1a8

Sociedad Geológica Mexicana. (2007). Carta Geológica Minera Autlan E13 - B12, Autlán de Navarro, Jalisco. Gobierno de Mexico. Edición de Cartas Geológico-Mineras y Geoquímicas. http://www.sgm.gob.mx/cartas/Cartas_Ed50. jsp

Vilches Vera, A. (2015). Resumen ejecutivo proyecto minero Mar y Cielo. Sernageomin. https://docplayer.es/14827257Resumen-ejecutivo-proyecto-minero-mar-y-cielo-drcs-antoniovilches-vera-ingeniero-civil-minas-peritomensurador-sernageomin.html

Yrarrazaval Herrera, M. J. (2013). Nueva Fórmula de Resistencia para el Diseño Empírico de Pilares de Roca [Universidad de Chile, Facultad de Ciencias Físicas y Matemáticas Departamento de Ingeniería de Minas]. http://repositorio. uchile.cl/handle/2250/113405 